\title{
The Incidence of Dysarrhythmias, Their Types and Outcomes in Patients Hospitalized with COVID -19
}

\author{
Abdulsatar Kamil Faeq (MRCP (UK)-MRCP (LONDON), HD cardiology) ${ }^{1}$ \\ ${ }^{1}$ College of Medicine, Hawler Medical University, Erbil, Iraq \\ email: awashor@yahoo.com
}

Received: 23 May 2021

Accepted: 29 June 2021

Published: 25 December 2021

Diyala Journal of Medicine 2021:21(2): 41-51

\begin{abstract}
Background: COVID-19 infection started in China and became a pandemic, the SARSCoV-2 virus mainly affects the respiratory system but can insult the cardiovascular system as well, higher rate of cardiac arrhythmias noted during the pandemic, and many studies showed that COVID-19 (especially hospitalized patients) can develop different types of arrhythmias which cause higher mortality rate.

Objective: To assess the incidence of dysrhythmias and their types and outcomes in hospitalized patients with COVID-19 in Erbil city.

Patients and Methods: Patients with documented COVID-19 infection admitted in Erbil Teaching Hospital respiratory care unit and coronary care unit from 1-8-2020 to 30-10-2020 had been included (255 patients) in this cross-sectional study, demographic data, and outcomes were reported, the ECG analyzed by cardiologists and the type of arrhythmia documented.

Results: Mean age was $47 \pm 12$ years, $61 \%$ of the patients were male, $25 \%$ of them had previous cardiac diseases, $20 \%$ diabetes, $16 \%$ multiple comorbidities, $8 \%$ hypertension, $8 \%$ obesity and $23 \%$ had no comorbidities, $20.7 \%$ of in-hospital patients developed dysrhythmias, $5.9 \%$ of them developed sinus tachycardia, $4.7 \%$ atrial fibrillation, $3.9 \%$ ventricular premature contractions, $2 \%$ ventricular fibrillation, $1.9 \%$ ventricular tachycardia and $1.9 \%$ heart blocks. Most of the patients who developed ventricular arrhythmias, atrial fibrillation, and heart block had previous comorbidities $82 \%, 62 \%$, and $80 \%$ respectively. Arrhythmias caused a higher in-hospital mortality rate $(39.6 \%$ versus $21.7 \%$ ) especially among male patients (mortality rate in male patients $43 \%$ compared with female patients $33 \%$ ).

Conclusion: Most of the admitted cases were male. Most of them had comorbidities especially previous cardiac diseases. Sinus tachycardia, atrial fibrillation, and ventricular extra-systoles were the most frequent arrhythmias. The mortality rate was increased by arrhythmias mainly in male patients.
\end{abstract}

Keywords: COVID-19, Dysrhythmias, Outcomes, Pandemic 
DOI: https://doi:10.26505/DJM.21026050523 , CAuthors, 2021, College of Medicine, University of Diyala.

This is an open access article under the CC BY 4.0 license (http://creativecommons.org/licenses/by/4.0/)

\section{Introduction}

Coronaviruses are important pathogens in both animals and humans, the novel coronavirus COVID -19, started in Wuhan city in Hubei Province in China rapidly became a pandemic and led to a considerable number of mortality [1].

Although respiratory system involvement is the most common manifestation of SARSCoV-2 infection, there are well documented cardiac insults in form of myocarditis, heart failure, acute coronary syndrome, and pericarditis [2].

Dysrhythmias generally can occur with many viral infections; the possible mechanisms for arrhythmogenicity are altered intercellular coupling, interstitial edema, and cardiac fibrosis which can lead to abnormal conduction in addition to abnormal $\mathrm{Ca} 2+$ handling and down-regulation of $\mathrm{K}+$ channels those results in repolarization abnormalities and action potential conduction abnormalities [3, 4].

Direct viral insult to the myocardium causing inflammation,, and through interaction with angiotensin-converting enzyme 2 resulting in its down-regulation and loss of its cardio-protective effect in addition to electrolyte abnormalities and QT prolongation are among factors resulting in arrhythmias in COVID-19 patients [4].

Although the underlying pathophysiology is elusive, various single-center studies and surveys around the world have reported a spectrum of dysrhythmias associated with the disease and its therapy, mainly atrioventricular heart block, atrial fibrillation, and polymorphic ventricular tachycardia [5].

Coexisting hypoxia, electrolyte abnormalities, and the administration of arrhythmogenic drugs (hydroxychloroquine and azithromycin) lead to great difficulty in ascertaining the direct and indirect roles of SARS-CoV-2 in the reported arrhythmias [4]. The increased number of hospitals cases of cardiac arrest in different parts of the world, (58\% increased rate in Lombardy in Italy and $52 \%$ in France) raised the point of the role of the virus in causing life-threatening arrhythmias [6].

In the United States, a large single-center study of 700 patients showed that admission to the intensive care unit was independently associated with a 10-fold increase in arrhythmia risk [6]. Cardiac arrest was associated with asignificant number of mortality in the hospitals; however nonshockable rhythms, atrial fibrillation, bradycardia, and non-sustained ventricular tachycardia were not associated with mortality [7].

There were different figures about the incidence of arrhythmia in hospitalized patients in different countries, in China (Wuhan) 25 out of 123 cases, in Iran (Tehran) 6 out of 25 cases, San Luca Hospital in Italy 34 out of 137 cases and in USA (New York Colombia University Medical center) 227 out of 1311 cases developed arrhythmias [8]. 
Some cohorts reported different types of arrhythmia in COVID 19 patients in around $7.3-17 \%$ in general cases and $44 \%$ of hospitalized patients [9].

Different percentages of dysrhythmias were reported in different centers, 133 cases of cardiac arrest were reported in patients with severe pneumonia in China, $13 \%$ of them resuscitated and remain alive, in another registry in 143 patients $15 \%$ ventricular ectopics, $1.4 \%$ ventricular fibrillation, $0.7 \%$ ventricular tachycardia and $1.4 \%$ bradyarrhythmias reported [10].

The aim of this study is to assess the incidence of dysrhythmias, their types, and outcomes in hospitalized patients with COVID-19 in Erbil city.

\section{Patients and Methods}

\section{Study design}

The study is a single-center cross-sectional study that included patients with COVID-19 admitted to CCU and RCU in Erbil Teaching Hospital for (255 patients), from 1-8-2020 to 30-10-2020.

Inclusion criteria: All in-hospital patients with clinical symptoms of COVID-19, who were hypoxemic either on face mask oxygen, noninvasive or invasive ventilation with either.

\section{1-CR test positive for SARS-CoV-2}

2-Positive for the specific $\operatorname{IgM}$ antibody and IgG antibody to SARS-CoV-2 in serum test 3-Change of the SARS-CoV-2-specific IgG antibody from negative to positive ranging from 8-77 years, the median age was 45.5 years, male: female ratio was 1.07:1.
4-Titer rising $\geq 4$ times in the recovery phase above that in the acute phase [11].

Exclusion criteria: Patients who didn't meet the inclusion criteria features.

Data collection: Demographic data including patient's age, gender, comorbidities reported, the ECG of the patients were interpreted, the arrhythmias diagnosis were made by cardiologists and the patients'outcome whether recovered and discharged from hospital, or died in the hospital were reported.

\section{Statistical analysis}

The data were shown in pie graph and analyzed by Microsoft excel program, using Chi-square test and P-value of 0.05 or less regarded as significant.

\section{Results}

The sample size included 255 patients, mean age was $47 \pm 12$ years, Male/Female ratio was $155 / 100$ (61\% were male). A highly significant number of the patients were having comorbidities (195 patients) accounts for $76.4 \%$, p-value $=0.0002$, diabetes mellitus present in 50 patients $(20 \%$ of the total number of patients), 20 patients had hypertension (8\%), 20 patients with obesity (8\%), 65 patients with a history of ischemic heart disease or heart failure $(25 \%), 40$ patient with multiple comorbidities) (16\%) and 60 patients had no comorbidities (23\%) as shown in figure (1), the differences among the groups are highly significant $\mathrm{p}$ value $=0.0001$. 


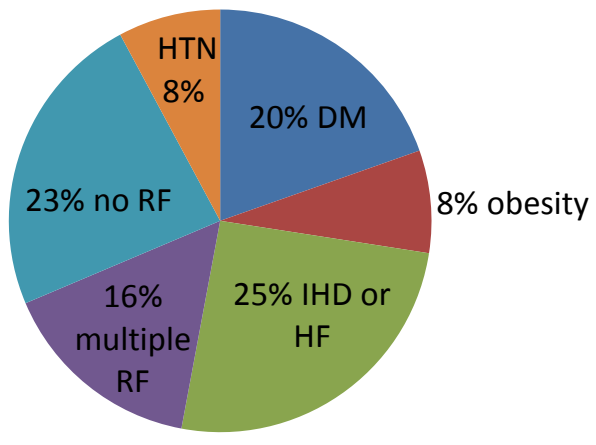

* HTN: Hypertension, DM: Diabetes mellitus, IHD: Ischemic heart disease, HF: Heart failure, RF: Risk factors (comorbidities)

Figure (2): Distribution of case according to the comorbidities

53 out of 255 patients (20.7\%) had and 9.4\% of those with arrhythmias), 5 documented dysrhythmias, 15 of them had patients with sustained ventricular severe sinus tachycardia (5.9\% of total cases tachycardia that had been successfully and $28 \%$ of those with arrhythmias), 12 cardioverted (1.9\% of total cases and $9.4 \%$ of patients with $\mathrm{AF}$ (4.7\% of total cases and those with arrhythmias) and 6 patients $22.6 \%$ of those with arrhythmias), 10 patients ventricular fibrillation (2\% of total cases and with ventricular premature beats (3.9\% of $11.3 \%$ of those with arrhythmias), two of total cases and $18.8 \%$ of those with them cardioverted successfully as shown in arrhythmias), 5 patients with second and Figure (2), the difference is not significant pthird-degree heart block $(1.9 \%$ of total cases value $=0.08$.

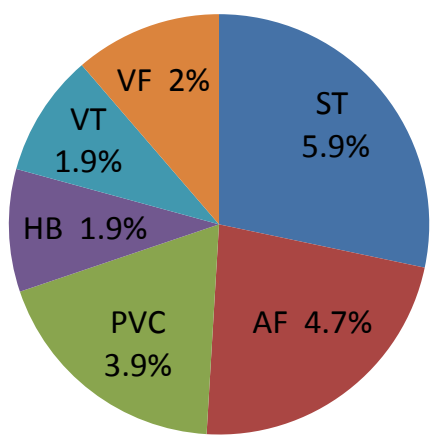

*ST: Sinus tachycardia, AF: Atrial fibrillation, PVC: Premature ventricular complex, HB: Heart block, VT: Ventricular tachycardia, VF: Ventricular fibrillation

Figure (2): Distribution of cases according to the types of dysrhythmias 
Among patients with ventricular tachycardia or ventricular fibrillation, 9 out of 11 patients $(82 \%)$ of them had a previous history of ischemic heart disease or heart failure (pvalue $=0.03)$, those patients with atrial fibrillation, 8 out of $12(67 \%)$ of them had either hypertension, diabetes or combined comorbidities $(p$-value $=0.08)$, those with bradarrhythmias 4 out of $5(80 \%)$ patients had previous heart failure $(\mathrm{p}$-value $=0.04)$, while only 5 out of 10 patients $(50 \%)$ with ventricular extra-systoles had comorbidities, and 5 out of 15 patients $(33 \%)$ of patients with sinus tachycardia had previous comorbidities $(\mathrm{p}$-value $=0.06)$ as shown in Figure (3).

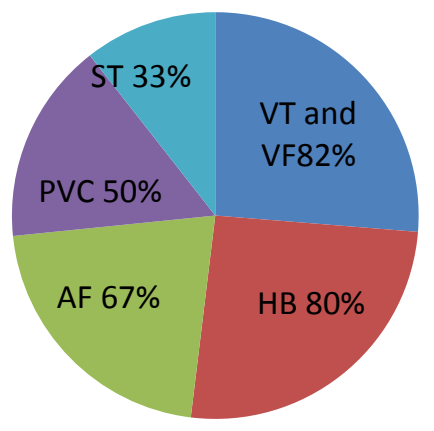

*ST: Sinus tachycardia, AF: Atrial fibrillation, PVC: Premature ventricular complex, HB: Heart block, VT: Ventricular tachycardia, VF: Ventricular fibrillation

Figure (3): Percentages of comorbidities in different types of dysrhythmias

The mortality rate among patients without shown in Figure 4, most of the deaths arrhythmias was $21.7 \%$, while among those occurred in patients who had ventricular with arrhythmia $39.6 \%$ (p-value $=0.02$ ), as tachyarrhythmia.

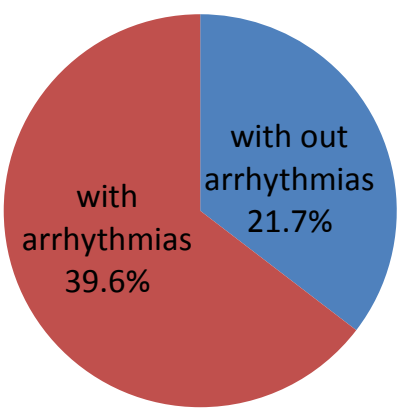

Figure (4): Mortality rate in patients with and without arrhythmias

The mortality rate in male patients with arrhythmias were $43 \%$ while in female it was
$33 \%$ (p-value $=0.009)$, as shown in Figure (5). 


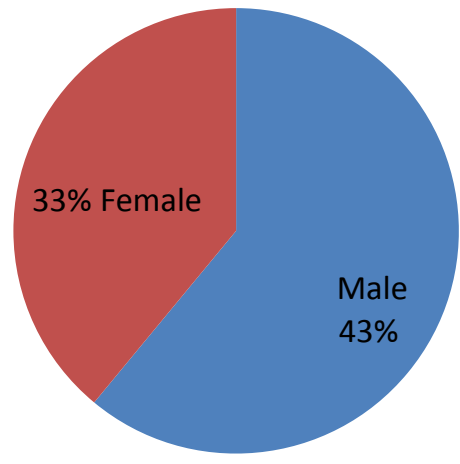

\section{Discussion}

Many studies showed that male patients are admitted to hospitals more than female patients Guido et al showed that admitted patients to intensive care units were male in $62 \%$ [12], Nanshan et al showed that $67 \%$ of infected patients were male [13], Borges et al in a meta-analysis $60 \%$ of admitted patients were male [14], Hannah P et al shown that males had three times higher odds for admission to the intensive care unit [15]. In this study, $61 \%$ of admitted cases were male; the differences with other studies were not significant ( $\mathrm{p}$-value 0.09). This gender effect was explained possibly by the ability of androgen to regulate transmembrane protease serine which is the enzyme that allows the final interaction between ACE and SARSCoV-2 virus [16, 17], this effect also had been noticed in animal models in mice as shown by Channaappanavar et al [18].

In many studies hypertension was the most prevalent comorbidities, Jing Y et al showed in a meta-analysis that hypertension was present in $21.1 \%$ of admitted patients [19], Ashkan et al showed that hypertension was present in $21 \%$ of moderate to severe cases [20], Wren et al in the review of two articles showed that hypertension is the most prevalent comorbidities [21], Kalpanta et al reviewed 27 articles including 22753 patients and found that hypertension was present in $27.4 \%$ of cases [22], the current study showed that hypertension present in only $8 \%$ of admitted cases, the differences was statistically significant ( $p$ value $=0.04$ ). In this study cardiovascular diseases (ischemic heart disease and heart failure) and diabetes mellitus were the most prevalent comorbidities $25 \%$ and $20 \%$, respectively, the prevalence of these two comorbidities were $8.4 \%$ and $9.7 \%$ respectively by Jing et al [19], the differences were statistically significant ( $\mathrm{p}$-value=0.05), Ashkan et al showed that diabetes present in $11 \%$ and cardiovascular diseases in $2.4 \%$ [20], the differences were statistically significant for cardiovascular disease $(\mathrm{p}$-value $=0.01)$ but not for diabetes ( $\mathrm{p}$-value $=0.09$ ), Kalpanta et al found cardiovascular diseases in $8.9 \%$ and diabetes in $17.4 \%$ [22], the differences were statistically significant for cardiovascular disease $(p$-value $=0.04)$ but not for diabetes (p-value=0.6). Kalpanta et al also showed major comorbidity specific to different 
countries; in South Korea, cardiovascular diseases were the most prevalent in $25.6 \%$ which is compatible with the current study $25 \%$, in Iran diabetes was the most prevalent comorbidity $35 \%$, which is statistically significant if compared with this study $20 \%$ (p-value $=0.03)$, in all other countries China, Italy, USA and UK hypertension was the most prevalent comorbidity 39.5\%, 35.9\%, $38.9 \%$ and $27.8 \%$ respectively, this study showed that hypertension is prevalent in $8 \%$ the differences were statistically highly significant $(\mathrm{p}$-value $=0.002)$.

In this study, dysrhythmias were documented in $20.7 \%$ of cases, Shu-Chen et al showed in a meta-analysis that the overall incidence of arrhythmia in COVID-19 patients was $16.8 \%$ [6] which is statistically not significant compared with the current study ( $\mathrm{p}$-value $=0.5$ ), Jae Hyung et al showed in a prospective study on 143 patients that the overall in-hospital incidence of arrhythmia was $25.2 \%$ [23] which is statistically not significant compared with current study $20.7 \%$ ( $p$-value $=0.55$ ), Irma et al showed in a pooled analysis that arrhythmia was quite prevalent in COVID-19, which was about $19 \%$ [24], which is statistically not significant compared with the current study $20.7 \%$ (p-value $=0.53$ ), in a study by Wang et al on 138 admitted COVID-19 patient in Wuhan $44 \%$ of them had arrhythmias [24], which is statistically highly significant compared with current study $20.7 \%$ (pvalue $=0.004)$.

In this study sinus tachycardia was seen in $28 \%$, atrial fibrillation in $22.6 \%$, premature ventricular complexes in $18.8 \%$, heart block in $9.8 \%$, sustained ventricular tachycardia in $9.8 \%$, and ventricular fibrillation in $11.2 \%$ of patients who developed arrhythmias, ShuChen et al showed that atrial fibrillation present in $8.2 \%$ of patients with arrhythmias [6], the difference is statistically highly significant compared with current study ( $p$ value $=0.009)$, he also showed that heart block seen in $10.8 \%$ of patients who developed arrhythmias [6] the difference is statistically not significant ( $p$-value $=0.5)$, premature ventricular contractions seen in $8.6 \%$ [6] the difference is statistically is significant $\quad(p$-value $=0.05)$, sustained VT reported in $3.3 \%$ [6] while in our study it was reported in $9.8 \%$ the difference is statistically is significant ( $p$-value $=0.05$ ). Jae Hyung et al showed that it was present in $39.9 \%$ of patients with arrhythmias [23], the difference is statistically not significant ( $\mathrm{p}$-value $=0.1$ ), premature ventricular complex in $28.7 \%$ [23] the difference is statistically not significant ( $\mathrm{p}$-value $=0.14$ ), while sustaining ventricular tachycardia seen in $1.4 \%$ [23], when compared with this study $9.8 \%$ the difference is statistically significant ( $p$-value $=0.01$ ). Irma et al showed in a pooled analysis that AF was the most frequent type $36 \%$ [24], his result when compared with the current study, $22.6 \%$ is statistically not significant (pvalue $=0.06$ ), in a study by Chun $\mathrm{JH}$ et al included 143 patients, non-sustained VT occurred in $15.4 \%$ when compared with this study $9.8 \%$ the difference is statistically not significant ( $\mathrm{p}$-value $=0.26$ ) and he reported premature ventricular contractions in $28.8 \%$ of patients when compared with this study $18.8 \%$ the difference is statistically not significant (p-value $=0.14)$ [26].

Halley et al mentioned in a review that the most prevalent comorbidities among patients with COVID-19 and arrhythmia were 
hypertension, history of myocardial infarction and diabetes [27] this study showed that cardiovascular diseases including ischemic heart disease and heart failure were the main comorbidity in COVID-19 patients who developed ventricular fibrillation, sustained ventricular tachycardia, heart block, and atrial fibrillation.

This study showed a significantly higher mortality rate among COVID-19 patients with arrhythmias especially ventricular arrhythmias. Raymond et al showed in a meta-analysis that included 784 patients that arrhythmias in COVID-19 patients had significantly poor outcome [28]. CorralesMedina et al showed that mortality rates in COVID-19 patients were higher among those with ventricular tachyarrhythmia [7] his results are compatible with this study. KarlHeinz in a review mentioned a high mortality rate among COVID-19 patients with malignant arrhythmias compared with others without [29], this study most of the deaths in patients with arrhythmia were driven biventricular tachyarrhythmia.

In a meta-analysis by Borges $\mathrm{N}$ et al which included 59000 patients they showed a higher mortality rate in male patients even in comparison to postmenopausal females [14], this is compatible with the results of the current study. Anta et al in a review mentioned that the mortality rate is higher in males patients with COVID-19 in most of the countries registries up to $3.5 \%$ higher rate have been reported, however, this study reported a higher rate in male up to $10 \%$, the difference is statistically significant ( $p$ value $=0.04$ ), however in India opposite results recorded, mortality was $3.3 \%$ in women and $2.9 \%$ in men [30]. Federico et al showed in an observational study that 28 days mortality in males was $38.1 \%$ and in women was $26.1 \%$ with difference of $12 \%$ [31]. This study showed a difference of $10 \%$ the result is statically not significant ( $p$ value $=0.5$ ).

\section{Conclusions}

Most of the hospitalized COVID-19 patients were male; most of the patients had comorbidities, the incidence of dysrhythmias was $20.7 \%$, the most frequent arrhythmia was sinus tachycardia followed by atrial fibrillation then ventricular extrasystoles, ventricular fibrillation, ventricular tachycardia and then heart block, arrhythmias were associated with higher mortality rate especially in male patients.

\section{Recommendations}

It's recommended to perform studies on cardiac arrhythmias in all COVID-19 centers in Iraq to have a higher sample size and impressive results.

Source of funding: This research was funded by ourselves and these is no other funding cover this study or manuscript preparation and publication.

Ethical clearance: Ethical approval for this study was issued by ethical committee of the College of Medicine-Hawler Medical University.

\section{Conflict of interest: Nill}

\section{References}

[1]Anjali B, Michael M , Srinath A , Matthew H , Eric Oh , Ann T et al. COVID19 and cardiac arrhythmias. Heart Rhythm 2020; 17(9): 1439-44.

[2] Ellie J, Stephanie K, Isaac G, Angelo B, Hasan G, Seth G et al. Worldwide Survey of 
COVID-19-Associated

Circulation 2020; 14(3): 25-9.

[3] Stephanie K, Ellie C, Ani N, Lauren R, Aakriti G, Mina $\mathrm{C}$ et al. Cardiac arrhythmias in COVID-19 infection. Circulation 2020; 13(6): 30-35.

[4] Parinita D, Joshua L, Pierre Q, Blake O, William H, Bruce $\mathrm{K}$ et al. Arrhythmia and COVID-19: A Review. JACC 2020; 6(9): 1193-204.

[5] Yueying W, Zhaojia W, Gary T, Lin Z, Elaine Y, Yutao G, et al. Cardiac Arrhythmias in patients with COVID-19. Journal of Arrhythmia 2020; 36(5): 827-36.

[6] Shu-Chen L, Shih-Chieh S, Chi-Wen $\mathrm{C}$,Yung-Chang $\mathrm{C}$ and Ming-Jui H. Incidence rate and clinical impacts of arrhythmia following COVID-19: a systematic review and meta-analysis of 17435 patients. Critical care 2020;10(6):125-32.

[7] Corrales-Medina VF, Kathryn N, Suh $\mathrm{KN}$, Rose G, Steve D, William $\mathrm{K}$ et al. Cardiac complications in patients with community-acquired pneumonia: a systematic review and meta-analysis of observational studies. June 28 2011; retrieved from

https://journals.plos.org/plosmedicine/article? id=10.1371/journal.pmed.1001048.

[8] Kang Y, Chen T, Mui D, Victor F, Dinesh J, Yushi $\mathrm{H}$ et al. Cardiovascular manifestations and treatment considerations in covid-19. Heart. 2020; 106(15):1132-41

[9] Driggin E, Madhavan MV, Bikdeli B, Taylor C, Justin L, David A et al. Cardiovascular considerations for patients, health care workers, and health systems during the COVID-19 pandemic. J Am Coll Cardiol. 2020; 75(18):2352-71.
[10] Guo T, Fan Y, Chen M, Xiaoyan N, Lin z, Tao $\mathrm{H}$ et al. Cardiovascular implications of fatal outcomes of patients with coronavirus disease 2019 (COVID-19). JAMA Cardiol. 2020;5(7):1-8. [11] Yun-Yun W, Ying-Hui J , Xue-Qun R, Yi-Rong L, Xiao-Chun Z, Xian-Tao Z et al. Updating the diagnostic criteria of COVID19 "suspected case" and "confirmed case" is necessary. June 2020; retrieved from https://mmrjournal.biomedcentral.com/track/ pdf/10.1186/s40779-020-00245-9.pdf

[12] Guido L, Guido G, Claudio B, Stefano $\mathrm{C}$, Francesco F, Claudio $\mathrm{F}$ et al. Gender differences in predictors of intensive care units admission among COVID-19 patients: The results of the SARS-RAS study of Italian Society of Hypertension. October 2020; doi:10.1371/journal.pone.0237297.Retrievedf romhttps://pubmed.ncbi.nlm.nih.gov/330220 04.

[13] Nanshan C, Min Z, Xuan D Jieming Q, Fengyun G, Yang $\mathrm{H}$ et al. Epidemiological and clinical characteristics of 99 cases of novel corona virus pneumonia in Wuhan, China: A descriptive study. Lancet 2020: 467-55.

[14] Borges N, Cacic A, von Groote TC, Thilo C, Umesh J, Ishanka $\mathrm{W}$ et al. Novel Coronavirus Infection (COVID-19) in Humans: A Scoping Review and MetaAnalysis. J Clin Med. 2020; 9(4): 45-54.

[15] Hannah P, Nina M, Charles R, Anna R, Coziana C, Lucy R et al. Male sex identified by global COVID-19 meta-analysis as a risk factor for death and ITU admission. $9^{\text {th }}$ December 2020; Retrieved from https://www.nature.com/articles/s41467-02019741-6. 
[16] Chen Z, Song X, Li Q et al. Androgen Receptor-Activated Enhancers Simultaneously Regulate Oncogene TMPRSS2 and IncRNA PRCAT38 in Prostate Cancer. Cells. 2019;8(8): 120-8.

[17] Bertram S, Glowacka I, Muller M, Lavender H, Gnirrs K, Nehlmeier I et al. Cleavage and activation of the severe acute respiratory syndrome coronavirus spike protein by human airway trypsin-like protease. J Virol. 2011;85(24):13363-72.

[18]Channappanavar R, Fett C, Mack M, Ten Eyck PP, Meyerholz DK, Perlman S. SexBased Differences in Susceptibility to Severe Acute Respiratory Syndrome Coronavirus Infection. J Immunol. 2017;198(10):404653.

[19] Jing Y, Ya Z, Xi G, Ke P, Chen Z, Guo $\mathrm{Q}$ et al. Prevalence of comorbidities and its effects in patients infected with SARS-CoV2: A systematic review and meta-analysis. Int $\mathrm{J}$ Infect Dis 2020; 94:91-5.

[20] Ashkan B, Muhammad H, Aslan B and Amir R. Prevalence of Comorbidities in COVID-19 Patients: A Systematic Review and Meta-Analysis. Arch Bone Jt Surg 2020; 8(1): 247-55.

[21]Wern H, Thomas T, Nigel M, Vermeulen J, Geodhals D, Joseph B et al. Comorbidities in SARS-CoV-2 Patients: a Systematic Review and Meta-Analysis. May 2020; doi: 10.1016/j.ijid.2020.03.017. Retrieved from https://mbio.asm.org/content/12/1/e03647-

20.

[22]Kaplanta B, Sujan B, Bishnu B and Maria J. Prevalence of comorbidities among individuals with COVID-19: A rapid review of current literature. AJIC 2021; 49:238-46. [23] Jae Hyung C, Ali N, Richard S, Archana R, Ashkan E, Michel $\mathrm{S}$ et al. Cardiac arrhythmias in hospitalized patients with COVID-19: A prospective observational study in the western United States. 28th December 2020; doi: 10.1371/journal.pone.0244533. Retrieved from https://pubmed.ncbi.nlm.nih.gov/33370347/. [24] Eka P, Irma M, Dita A and Rerdin J. Atrial arrhythmia and its association with COVID-19 outcome. 19th January 2021. Retrieved from https://www.degruyter.com/document/doi/10. 1515/dx-2020-0155/html.

[25] Wang D, Hu B and Hu C. Clinical Characteristics of 138 Hospitalized Patients With 2019 Novel Coronavirus-Infected Pneumonia in Wuhan, China. JAMA 2020; 323(11): 1061.

[26] Shabo Shi, Mu Quin and Bo Shen . Association of Cardiac Injury with Mortality in Hospitalized Patients with COVID-19 in Wuhan, China. JAMA Cardiol.2020;5(7):802-10.

[27]Halley $\mathrm{H}$ and Adel E. Cardiac arrhythmias in COVID-19 patients: A combination of viral comorbidities and proarrhythmic drug interactions. Am J Emerg Med 2021; Retrieved from https://www.ncbi.nlm.nih.gov/pmc/articles/P MC7847181/.

[28] Raymond P, Ian H and Sunu Budhi R. Incidence and impact of cardiac arrhythmias in coronavirus disease 2019 (COVID-19): A systematic review and meta-analysis. Indian Pacing Electrophsiol J 2020; 20(5):193-8.

[29] Karl-Heinz K. Arrhythmias and sudden cardiac death in COVID-19 pandemic. 2020; Retrieved from https://www.ncbi.nlm.nih.gov/pmc/articles/P MC7181098/. 
[30] Anita R and Nabamallika D. Sex differences in COVID-19 case fatality: Do we know enough? 2020; From https://www.thelancet.com/journals/langlo/ar ticle/PIIS2214-109X(20)30464-2/fulltext

[31] Federico R and Luca N. Covid-19 and gender: lower rate but same mortality of severe disease in women - an observational study. 2021; from https://bmcpulmmed.biomedcentral.com/artic les/10.1186/s12890-021-01455-0. 